\title{
ORGANIZAÇÃO DA ATENÇÃO PRIMÁRIA PARA DIAGNÓSTICO E TRATAMENTO DA TUBERCULOSE
}

\author{
Mariana César dos Santos ${ }^{1}$, Rayla Patrícia da Silva Andrade ${ }^{1}$, Sonaly Melo de Macedo ${ }^{1}$, Anny Suelen dos \\ Santos Andrade ${ }^{1}$, Tereza Cristina Scatena Villa², Érika Simone Galvão Pinto ${ }^{3}$
}

RESUMO: Objetivou-se descrever a percepção dos profissionais da Atenção Primária, atuantes no município de Natal, acerca da organização da atenção à tuberculose. Trata-se de um estudo exploratório, de abordagem quantitativa, realizado com 100 profissionais da Atenção Primária, entre novembro de 2013 e janeiro de 2014. Utilizou-se para coleta de dados instrumento estruturado. Dos resultados obtidos, $42 \%(n=42)$ dos profissionais afirmaram que o gerente da unidade de saúde faz parte do planejamento em relação à tuberculose; $43 \%$ ( $n=43)$ reconheceram as metas pactuadas como revistas periodicamente; $52 \%(n=52)$ confirmaram a existência de estratégias para a melhoria da atenção à tuberculose; $56 \%(n=56)$ alegaram a oferta de benefícios e incentivos aos portadores de tuberculose. Conclui-se que a capacidade organizativa está ligada às estratégias governamentais, as quais podem induzir o interesse do gestor a garantir a oferta de benefícios e evidenciar a Atenção Primária como local para o tratamento e diagnóstico da tuberculose.

DESCRITORES: Tuberculose; Atenção primária à saúde; Serviços de saúde; Assistência ao paciente.

\section{ORGANIZATION OF PRIMARY HEALTHCARE SERVICES FOR TUBERCULOSIS DIAGNOSIS AND TREATMENT}

ABSTRACT: The present study aimed to describe how Primary Care professionals, in the city of Natal, state of Rio Grande do Norte, perceive the organization of health care services for tuberculosis. Exploratory study, with a quantitative approach, carried out with 100 professionals of PHC, between November 2013 and January 2014. Data was collected with the use of a structured instrument. According to the results, $42 \%(n=42)$ of the professionals affirmed that health unit managers play a key role in the planning for tuberculosis care; $43 \%(n=43)$ reported that the agreed targets are periodically reviewed; $52 \%(n=52)$ confirmed the existence of strategies to improve care services for tuberculosis; $56 \%(n=56)$ mentioned the benefits and incentives offered to tuberculosis patients. It is concluded that the organizational capacity in primary care is related to governmental strategies aimed to ensure that health unit managers provide benefits and incentives to TB patients and that tuberculosis treatment is delivered at the primary care level.

DESCRIPTORS: Tuberculosis; Primary health care; Health services; Patient care.

\section{ORGANIZACIÓN DE LA ATENCIÓN PRIMARIA PARA DIAGNÓSTICO Y TRATAMIENTO DE LA TUBERCULOSIS}

RESUMEN: La finalidad de este estudio fue describir la percepción acerca de la organización de la atención a la tuberculosis de los profesionales de la Atención Primaria que actuan en el municipio de Natal. Es un estudio exploratorio, de abordaje cuantitativo, realizado con 100 profesionales de la Atención Primaria, entre noviembre de 2013 y enero de 2014. Para obtener los datos, se utilizó instrumento estructurado. Como resultado, se verificó que $42 \%(n=42)$ de los profesionales afirmaron que el gerente de la unidad de salud hace parte del planeamiento acerca de la tuberculosis; $43 \%(n=43)$ reconocieron las metas acordadas como siendo revistas periodicamente; $52 \%(n=52)$ confirmaron la existencia de estrategias para mejorar el proceso de la atención a la tuberculosis; $56 \%$ $(n=56)$ argumentaron que hay oferta de incentivos y benefícios a los portadores de tuberculosis. Se constata que la capacidad de organización está enlazada con las estrategias de gobierno, las cuales pueden inducir motivar el gestor a garantizar la oferta de beneficios y evidenciar la Atención Primaria como proceso para el tratamiento y diagnóstico de la tuberculosis.

DESCRIPTORES: Tuberculosis; Atención primaria a la salud; Servicios de salud; Asistencia al paciente.

${ }^{1}$ Discente de Enfermagem. Universidade Federal do Rio Grande do Norte. Natal, RN, Brasil.

${ }^{2}$ Enfermeira. Doutora em Enfermagem. Docente da Escola de Enfermagem de Ribeirão Preto. Ribeirão Preto, SP, Brasil.

${ }^{3}$ Enfermeira. Doutora em Enfermagem. Docente da Universidade Federal do Rio Grande do Norte. Natal, RN, Brasil.

Autor Correspondente:

Rayla Patrícia da Silva Andrade

Universidade Federal do Rio Grande do Norte

Av. Senador Salgado Filho 3000 - 59078970 - Natal, RN, Brasil

Email: raylapatriciayla@gmail.com
Recebido: $17 / 08 / 2016$

Finalizado: 22/05/2017 


\section{- INTRODUÇÃO}

A tuberculose (TB) é considerada como doença de incidência mundial e grave problema de saúde pública. Em 2015, 6,1 milhões de novos casos de TB foram notificados à Organização Mundial da Saúde (OMS). De acordo com a nova classificação da OMS 2016-2020, o Brasil ocupa a $20^{a}$ posição na lista dos 30 países com alta carga de TB e a 19a posição na lista dos 30 países com alta carga de TB/HIV ${ }^{(1)}$.

No contexto epidemiológico, a TB é considerada prioridade e está contemplada nos programas desenvolvidos pelo Ministério da Saúde (MS), por meio de ações de controle, prevenção e vigilância da doença ${ }^{(2)}$.

Dentre os programas de ações e serviços, destaca-se o Plano Nacional de Controle à Tuberculose (PNCT), desenvolvido nas três esferas do governo, com maior ênfase na esfera municipal, no nível da Atenção Primária à Saúde (APS), com as atividades de prevenção e promoção(3).

O Sistema Único de Saúde (SUS) decreta como porta de entrada aos serviços de saúde a APS, o Pronto Atendimento e outros serviços de saúde ${ }^{(4)}$. A APS merece destaque, uma vez que dispõe de equipes da Estratégia Saúde da Família (ESF), que desenvolvem ações de busca ativa de pacientes suspeitos, notificação de casos, tratamento, acompanhamento e alta comprovada por cura, além de ações preventivas de educação em saúde ${ }^{(3)}$.

A incidência de TB não está isoladamente relacionada a fatores como subdesenvolvimento socioeconômico ou demais fatores de vulnerabilidade ao adoecimento, sendo a fragilidade estrutural e de organização da gestão dos serviços de saúde também contribuintes para o aumento desses índices ${ }^{(2)}$. A APS vem se fortalecendo desde a criação do $S U S^{(5)}$ e a implementação dos pactos à saúde, com metas e indicadores estabelecidos aos municípios e estados, que recebem recursos financeiros e estabelecem responsabilidades entre os gestores em prol do acesso da população aos serviços de saúde $^{(6)}$.

A organização do serviço de saúde é definida como a forma do desenvolvimento da divisão técnica e social do trabalho em saúde, onde as práticas e gestões profissionais e do cuidado se correlacionam ${ }^{(7)}$. É neste âmbito que o processo de trabalho é realizado, coordenando-se com as diversas formas de tecnologia em saúde ${ }^{(6)}$.

Os motivos que conduziram a realização desse estudo foram a vivência no grupo de pesquisa em TB e o interesse despertado durante reunião técnica acerca da organização da rede assistencial da TB no município de Natal, estado do Rio Grande do Norte.

O estudo se faz relevante para os profissionais da APS no que se refere a identificar lacunas na organização do serviço e contribuir para a melhoria da assistência ao portador de TB. Diante desse contexto, o trabalho tem como objetivo descrever a organização da APS na assistência ao portador de TB, no município de Natal, na visão dos profissionais.

\section{METODOLOGIA}

Estudo quantitativo, transversal, realizado no município de Natal, onde se realizou a caracterização do atual cenário da TB.

A população foi composta por profissionais que já acompanharam casos de TB na APS, totalizando 384 profissionais. Utilizou-se amostra aleatória, considerando os parâmetros: Erro amostral de 0,5; Intervalo de Confiança de 95\% e P (proporção populacional) de 50\%. Obteve-se a amostra equivalente a 100 profissionais $(n=100)$.

O município de Natal tem suas ações de atenção e gestão desenvolvidas por cinco distritos sanitários - Norte I e II, Sul, Leste e Oeste. A área administrativa é delimitada conforme seu território de responsabilidade ${ }^{(8)}$.

Para otimização do controle e monitoramento da TB, Natal precisa monitorar e cumprir metas 
e indicadores estabelecidas pelo PNCT. São elas: manter a detecção anual de pelo menos $70 \%$ dos casos estimados de TB; tratar corretamente 100\% dos casos de TB diagnosticados e curar pelo menos $85 \%$ destes; manter o abandono de tratamento em percentuais considerados aceitáveis (5\%); manter registro atualizado dos casos diagnosticados e $100 \%$ do resultado de tratamento e disponibilizar teste anti-HIV para $100 \%$ dos adultos com TB. Quanto aos indicadores, destacam-se a taxa de cobertura vacinal com BCG ID em menores de um ano; número de sintomáticos respiratórios examinados; proporção de casos de TB pulmonar com baciloscopia realizada; proporção de bacilíferos entre o total de casos pulmonares; proporção de profissionais da APS capacitados em ações/atividades do PNCT; percentual de cura dos casos de tuberculose após nove meses; percentual de abandono de tratamento de tuberculose; percentual de óbitos ${ }^{(9)}$.

O período da coleta de dados ocorreu de novembro de 2013 a janeiro de 2014. O instrumento foi aplicado com quatro profissionais em cada unidade de saúde, sendo um por categoria (médico, enfermeiro, técnico em enfermagem e agente comunitário de saúde). Havendo rejeição de alguma categoria profissional, o instrumento foi aplicado com outro profissional de saúde das outras três categorias, de forma que totalizasse 04 por unidades de saúde. Os profissionais que aceitaram participar foram informados pelos pesquisadores quanto à pesquisa e os objetivos do estudo, mediante assinatura do Termo de Consentimento Livre e Esclarecido.

Utilizaram-se como critérios de inclusão: profissionais da APS que já acompanharam casos de TB, e de exclusão profissionais não atuantes no período de coleta de dados, que não tinham acompanhado portadores de TB durante o tratamento e que não aceitaram assinar o TCLE.

Utilizou-se um questionário estruturado baseado em uma proposta de MacCooll Institute for Health Care Innovation, Assessment of Chronic IIIness Care (ACIC) para a "avaliação da capacidade institucional local para desenvolver o modelo de atenção às condições crônicas" adaptado por Villa e Ruffino $^{(10)}$ para a avaliação das ações desenvolvidas para o controle da TB.

O instrumento foi dividido em sete dimensões, sendo a dimensão I (Organização da atenção à TB) o foco deste estudo. Esta dimensão teve como componentes: interesse do gerente na unidade de saúde em relação à TB; metas pactuadas e registradas pela unidade de saúde (cobertura de tratamento supervisionado e solicitação de exames de baciloscopia de escarro); estratégias para melhoria da atenção à TB (oferta de tratamento supervisionado, flexibilidade de horário do tratamento supervisionado, atividades educativas e prioridade no atendimento); estratégias para que a APS seja o local para tratamento da TB (facilidade de agendamento para atendimento, retaguarda laboratorial, capacitação dos profissionais na Unidade de Saúde); incentivo do gerente da unidade de saúde (se dá prioridade e incentiva esforços para a melhoria da atenção à TB) e benefícios e incentivos aos portadores de TB (café da manhã, leite, vale transporte e cesta básica).

As respostas foram divididas em quatro níveis ( $D, C, B, A)$, sendo o $D$ correspondente ao nível mais desfavorável, com pontuação de 0 a 2; nível $C$ de 3 a 5; nível $B$ de 6 a 8 (sendo os dois níveis intermediários) e nível A o mais favorável com pontuação de 9 a 11. A interpretação dos resultados deu-se da seguinte forma: capacidade limitada para a atenção aos portadores de TB (Pontuações de 0 a 2), capacidade básica para atenção aos portadores de TB (Pontuações de 3 a 5), capacidade razoável para a atenção aos portadores de TB (Pontuações de 6 a 8) e capacidade ótima para a atenção aos portadores de TB (Pontuações de 9 a 11). Calculado a partir da média aritmética da soma da pontuação de cada nível dividido pela quantidade de componentes.

O projeto foi encaminhado para o Comitê de Ética em Pesquisa da Universidade Federal do Rio Grande do Norte. Obteve parecer aprovado, mediante o número de protocolo $\mathrm{n}^{\circ} 456.332$ e data de registro em 01/11/2013. Para a realização deste estudo, respeitaram-se as Diretrizes e Normas Regulamentadoras de Pesquisas envolvendo seres humanos, resolução de $n^{\circ} 466$, de 12 de dezembro de 2012, do Conselho Nacional de Saúde(11).

A partir das variáveis do instrumento, os dados foram organizados, categorizados e codificados. A análise dos dados foi realizada utilizando o Statistical Package for Social Sciences (SPSS) versão 22.0. Utilizou-se a estatística descritiva, sendo a apresentação dos dados realizada por meio de tabelas. 


\section{Perfil dos profissionais de saúde}

Dentre os profissionais entrevistados, 34\% $(n=34)$ pertenciam à categoria de enfermeiro, $10 \%(n=10)$ de médico, 22\% ( $n=22)$ de técnicos em enfermagem e 34\% $(n=34)$ eram Agentes Comunitários de Saúde (ACS).

Quanto à caracterização da unidade de saúde em que os profissionais desenvolviam suas atividades durante a entrevista, $80 \%$ ( $n=80$ ) dos profissionais atuavam em Unidade de Saúde da Família (USF), 18\% ( $n=18)$ em Unidade Básica de Saúde (UBS) e dois (2\%) em Unidade Mista. No que tange ao tempo de serviço dos profissionais de saúde, sete $(7 \%)$ trabalhavam na unidade de saúde há menos de um ano, $23 \%(n=23)$ de um a cinco anos, $29 \%(n=29)$ de seis a dez anos e $41 \%(n=41)$ há mais de dez anos.

Em relação ao tempo que exerciam a função, três (3\%) dos profissionais estavam há menos de um ano no exercício da profissão, quatro ( $4 \%$ ) estavam de um a cinco anos, $14 \%(n=14)$ de seis a dez anos e $79 \%(n=79)$ a mais de dez anos.

\section{Classificação da organização da atenção à TB}

Sobre a organização da atenção à TB que engloba o manejo dos programas de controle, cobertura de tratamento, benefícios, incentivos e estratégias para melhoria do cuidado aos portadores da doença, $61 \%(n=61)$ das unidades de saúde foram classificadas como tendo capacidade razoável para a atenção aos portadores de TB, como mostra a Tabela 1.

Tabela 1 - Classificação da organização da atenção à tuberculose segundo a capacidade. Natal, RN, Brasil, 2014

\begin{tabular}{lcc}
\multicolumn{3}{l}{ Organização da Atenção a Tuberculose no Município de Natal } \\
\hline Capacidade & $\mathbf{N}$ & $\mathbf{\%}$ \\
\hline BÁSICA & 14 & 14 \\
\hline RAZOÁVEL & 61 & 61 \\
\hline ÓTIMA & 25 & 25 \\
\hline TOTAL & $\mathbf{1 0 0}$ & $\mathbf{1 0 0}$
\end{tabular}

\section{Classificação dos componentes da dimensão organização da atenção à tuberculose}

Os profissionais de saúde consideraram como capacidade ótima o componente relacionado ao interesse do gerente da unidade de saúde, o qual participa do planejamento da unidade com $42 \%$ $(n=42)$.

As metas pactuadas e registradas (cobertura de tratamento supervisionado e solicitação de exames de baciloscopia de escarro) pela unidade de saúde para o controle da TB na área de abrangência obteve $43 \%(n=43)$, sendo acompanhadas e revistas periodicamente e incorporadas no planejamento da unidade.

As estratégias para a melhoria da atenção à TB (oferta de tratamento supervisionado, flexibilidade de horário do tratamento, atividades educativas, prioridade no atendimento e facilidade de agendamento) com $52 \%(n=52)$, existem e são utilizadas para a prevenção de problemas.

Os benefícios e incentivos aos portadores de TB (café da manhã, leite, vale transporte e cesta básica) são ofertados para promover maior adesão dos pacientes com $56 \%(n=56)$.

Já como capacidade razoável foram considerados dois componentes: as estratégias para que a APS seja o local de tratamento da TB com $72 \%(n=72)$, no que se refere à facilidade de agendamento para atendimento, retaguarda laboratorial e capacitação dos profissionais na Unidade de Saúde, as quais 
existem para melhorar a assistência, promover maior acesso, vínculo e adesão ao tratamento; e o incentivo de esforços do gerente da unidade (identificação de portadores de TB na comunidade) para a melhoria da assistência à TB com 60\% $(n=60)$, conforme a Tabela 2.

Tabela 2 - Classificação dos Componentes da Dimensão Organização da Atenção à Tuberculose segundo a capacidade. Natal, RN, Brasil, 2014

\begin{tabular}{|c|c|c|c|c|c|c|c|c|c|c|}
\hline \multirow{3}{*}{$\begin{array}{l}\text { Componentes } \\
\text { Interesse do Gerente } \\
\end{array}$} & \multirow{2}{*}{\multicolumn{2}{|c|}{$\begin{array}{c}\text { Capacidade } \\
\text { LIMITADA } \\
\text { n } \%\end{array}$}} & \multirow{2}{*}{\multicolumn{2}{|c|}{$\begin{array}{c}\text { Capacidade } \\
\text { BÁSICA } \\
\text { n } \%\end{array}$}} & \multicolumn{2}{|c|}{$\begin{array}{l}\text { Capacidade } \\
\text { RAZOÁVEL }\end{array}$} & \multicolumn{2}{|c|}{$\begin{array}{l}\text { Capacidade } \\
\text { ÓTIMA }\end{array}$} & \multicolumn{2}{|c|}{$\begin{array}{l}\text { Capacidade } \\
\text { TOTAL }\end{array}$} \\
\hline & & & & & $\mathbf{n}$ & $\%$ & $\mathbf{n}$ & $\%$ & n & $\%$ \\
\hline & 12 & 12 & 8 & 8 & 38 & 38 & 42 & 42 & 100 & 100 \\
\hline Metas Pactuadas e Registradas & 8 & 8 & 16 & 16 & 33 & 33 & 43 & 43 & 100 & 100 \\
\hline $\begin{array}{l}\text { Estratégias para melhoria da atenção à } \\
\text { TB* }\end{array}$ & 7 & 7 & 8 & 8 & 33 & 33 & 52 & 52 & 100 & 100 \\
\hline $\begin{array}{l}\text { Estratégias para que a APS** seja o local } \\
\text { de tratamento da TB* }\end{array}$ & 3 & 3 & 4 & 4 & 72 & 72 & 21 & 21 & 100 & 100 \\
\hline $\begin{array}{l}\text { Incentivo do gerente da unidade de } \\
\text { saúde na assistência à TB* }\end{array}$ & 9 & 9 & 6 & 6 & 60 & 60 & 25 & 25 & 100 & 100 \\
\hline $\begin{array}{l}\text { Benefícios e incentivos aos portadores } \\
\text { de TB* }\end{array}$ & 2 & 2 & 11 & 11 & 31 & 31 & 56 & 56 & 100 & 100 \\
\hline
\end{tabular}

TB*- Tuberculose

APS**-Atenção Primária a Saúde

\section{- DISCUSSÃO}

Neste estudo, os dados do perfil dos profissionais de saúde quanto ao tempo de serviço corroboraram com estudo realizado em Ceará-Mirim-RN no ano de 2010 em que a maioria dos profissionais atuantes na APS está no serviço há mais de 10 anos. Essa experiência adquirida ao longo dos anos proporciona olhar abrangente sobre o usuário, possibilitando mudanças positivas em seu processo de trabalho ${ }^{(12)}$.

A classificação da organização da Atenção Primária aos portadores de TB, definida pela maioria dos profissionais de saúde como razoável, pode estar relacionada ao manejo de políticas e de programas de controle, como por exemplo, nas atividades de detecção e busca ativa de sintomáticos respiratórios. Essa organização pode ser mais efetiva se todos os profissionais da unidade estiverem preparados e qualificados. A capacitação profissional, quando realizada de forma periódica, pode garantir resultados satisfatórios no planejamento das ações e no plano de cuidados do paciente ${ }^{(13)}$.

A análise dos dados obtidos na pesquisa enfatiza o desenvolvimento do papel do gestor da unidade de saúde no âmbito municipal. Dos profissionais entrevistados, a grande maioria constatou o interesse do gerente da unidade em relação a sua participação durante o planejamento das ações a respeito da TB, juntamente com os profissionais.

A atuação do gerente da unidade de saúde vai além da organização do trabalho e gestão de recursos humanos. Presume-se autonomia e poder de decisão nas ações e nos serviços, para o desenvolvimento da assistência com qualidade por parte dos profissionais de saúde, no que diz respeito ao planejamento do cuidado ao portador de $\mathrm{TB}^{(14)}$.

Nesse contexto, evidenciam-se as metas e indicadores pactuados com capacidade ótima pela maioria dos entrevistados, tais como cobertura de tratamento supervisionado como fator a direcionar e influenciar o interesse do gestor local e instrumentos na realização das ações e atividades para o controle da TB. Aliado às metas e indicadores pactuados, outros recursos de avaliação dos resultados podem ser evidenciados como promotores do interesse do gestor local, uma vez que permite bônus/ recursos ao município de acordo com seu desempenho e cobra a prestação de contas do gestor à sociedade e outras esferas do governo ${ }^{(15)}$. 
Dentre os recursos, destacam-se: o Programa Nacional de Melhoria do Acesso e da Qualidade da Atenção Básica (PMAQ-AB) - que avalia o desempenho da unidade e qualificação do resultado das ações; o componente de qualidade do Piso de Atenção Básica Variável (PAB Variável) - recurso financeiro variável em função do desempenho obtido pelas equipes da APS; além dos relatórios gerenciais, implantado pelas Normas Operacionais Básicas da Saúde (NOBs), que permitem o controle e acompanhamento das ações no âmbito local ${ }^{(16)}$.

No componente que trata das metas pactuadas pela unidade de saúde (cobertura de tratamento supervisionado e solicitação de exames de baciloscopia de escarro) para o controle da TB na área de abrangência, os profissionais de saúde referiram acompanhamento e revisão periódica sendo incorporadas no planejamento da unidade.

Metas e indicadores pactuados fazem parte do processo de planejamento do SUS para as três esferas de governo e são classificadas em específicas, quando no âmbito local, e universal quando são de pactuação obrigatória ${ }^{(17)}$.

Deste modo, periodicamente é necessário reavaliar os indicadores e redirecionar ações, detectando obstáculos, propondo soluções, cronogramas e novas metas.

É importante também que os municípios realizem reuniões, pelo menos trimestrais, para avaliação das atividades do programa com seus serviços de saúde ${ }^{(18-19)}$.

Diante desse panorama delegado pelo MS, o resultado da entrevista não condiz quando se confronta com o atual cenário municipal. Dados de casos de TB obtidos sobre o município de Natal no ano de 2012 mostram a obtenção de desempenho abaixo do preconizado, ressaltando-se oferecimento de baciloscopia para $44,6 \%$ dos casos suspeitos, apesar de o preconizado ser $100 \%{ }^{(20)}$.

Tal resultado merece ser refletido enquanto capacidade dos profissionais de avaliar os serviços em que atuam, uma vez que fazem parte do processo de prestação destes serviços. Sugere-se capacitação contínua destes profissionais a fim de saberem identificar as preconizações dos programas de controle da TB e as lacunas existentes no sistema de saúde no qual estão inseridos.

Quanto às estratégias para a melhoria da atenção à TB, no que diz respeito à oferta de tratamento supervisionado, flexibilidade de horário do tratamento, atividades educativas, prioridade no atendimento e facilidade de agendamento, foi visto que existem e são utilizadas para a prevenção de problemas.

Fragilidades da organização dos serviços de saúde podem influenciar diretamente na satisfação do usuário destes serviços e, assim, sua procura e utilização ${ }^{(20)}$.

Um estudo que avaliou a satisfação dos portadores de TB quanto aos serviços de saúde entre os anos de 2008 e 2009 em um município paulista, evidenciou que fatores que afetam a dinâmica de vida - tempo de espera para consulta de controle - e o acesso do usuário à unidade de saúde - disponibilidade para consultas eventuais ou de urgência, relacionadas principalmente aos efeitos adversos do tratamento medicamentoso ou à piora dos sintomas - foram evidenciados pelos usuários como indicadores de satisfação com o serviço ${ }^{(21)}$. O que corrobora com os resultados desta pesquisa, quando considera as estratégias utilizadas como ferramentas primordiais para a melhoria da atenção à TB na APS.

Com relação às estratégias para que a APS seja o local para o tratamento da TB no que se refere à facilidade de agendamento para atendimento, retaguarda laboratorial, e capacitação dos profissionais, essas estratégias existem para melhorar a assistência ao portador de TB com o intuito de promover maior acesso, vínculo e adesão ao tratamento.

Apesar da APS se configurar como a porta de entrada de menor complexidade dentro das redes de serviços de saúde ${ }^{(22)}$, deve ser a preferencial para novos casos de TB $^{(19)}$, por dispor de ações e medidas dos programas governamentais - como ESF e Programa de Agentes Comunitários de Saúde (PACS) que estabelecem busca ativa e identificação dos sintomáticos respiratórios ${ }^{(23)}$.

A TB, por ser doença infectocontagiosa e o diagnóstico precoce ser a principal ação para o seu controle mediante a quebra da cadeia de transmissão(23), a APS se evidencia dentre os níveis de atenção. A literatura traz que o retardo no diagnóstico está ligado à demora da busca do tratamento, 
ressaltando o oferecimento da busca ativa e divulgação de informações como estratégias facilitadoras do diagnóstico ${ }^{(21)}$, o que torna a APS o local estratégico para o diagnóstico e tratamento da TB.

Uma pesquisa realizada no município de Natal no ano de 2012 com pacientes acometidos por TB pulmonar mostrou que a maioria dos entrevistados (56,7\%) optou pela procura dos serviços de urgência (hospitais e pronto atendimento), enquanto os demais $(43,3 \%)$ buscaram serviços eletivos (unidade de saúde e ambulatório de referência) como primeiro local para atendimento e diagnóstico da doença ${ }^{(23)}$. Tal resultado corrobora com os dados da pesquisa em questão, quanto à classificação razoável para a APS no município enquanto local para o tratamento da TB.

Os gerentes das unidades de saúde incentivam esforços para as mudanças na atenção à TB através do incentivo à identificação de portadores de TB na comunidade, como também dá prioridade à melhoria da assistência à TB.

A satisfação com o trabalho deve ser levada em consideração em seu contexto de melhoria da atenção à saúde, visto que o grau de satisfação do trabalhador reflete diretamente na forma de enfrentamento de problemas e na tomada de decisões ${ }^{(24)}$.

O gerente local deve atuar como interlocutor e mediador do processo de trabalho, em prol de atingir metas descritas no planejamento, sendo a formação do gestor direcionada para estratégias da APS - com evidência da ESF - assim como sua formação na área da saúde, recursos facilitadores desse processo de atuação(25).

Um estudo realizado em 2010 em São Paulo com gestores e especialistas da área da saúde, evidenciou que a formação em ciências da saúde e especialização em gestão de serviços de saúde foram as questões mais bem pontuadas quanto ao perfil para a seleção do gestor ${ }^{(26)}$.

Os benefícios e incentivos (café da manhã, leite, vale transporte e cesta básica) são ofertados para promover a melhora da atenção aos portadores de TB. Podem estar relacionados ao fato de que benefícios e incentivos são oferecidos como parte do PNCT, garantindo, entre outros, suporte nutricional ou de transporte ${ }^{(18)}$, num cenário em que há grande incidência de pessoas com déficit nutricional e em situações econômicas vulneráveis. Tais incentivos tendem a melhorar a adesão do paciente ao tratamento, tornando-se uma prática relevante ${ }^{(27)}$.

Durante as entrevistas, observou-se que o benefício prevalente é a cesta básica. Uma pesquisa realizada em 2006 e 2010 no município de Natal, com dados de abandono do tratamento da TB, tem-se um percentual que passou, respectivamente, de $22,73 \%$ para $6,49 \%$, evidenciando acentuada redução dos $\operatorname{casos}^{(28)}$.

Dentre as limitações do estudo, destaca-se a diferença do quantitativo de profissionais por categoria profissional, o que impede de fazer inferência à população por categorias.

\section{CONCLUSÃO}

O estudo permitiu conhecer a forma como está organizada a APS para o diagnóstico e tratamento da TB na visão dos profissionais.

Embora classificada como capacidade ótima, os componentes relacionados ao interesse do gerente da unidade de saúde, metas pactuadas e registradas pela unidade de saúde para o controle da TB na área de abrangência, estratégia para a melhoria da atenção à TB e benefícios e incentivos aos portadores de TB, obteve-se como capacidade razoável o incentivo de esforços para a melhoria da atenção à TB por parte do gerente da unidade de saúde.

Diante disso, faz-se necessário que os gestores de saúde utilizem os planos e estratégias implementadas como ferramentas a serem monitoradas e analisadas, podendo assim denotar seu interesse como líder situacional, na visão dos profissionais que compõem sua equipe multiprofissional, e assim contribuir para mudar o cenário da TB em Natal.

Sugere-se capacitação continuada aos profissionais de saúde com o intuito de situá-los quanto à importância do alcance das metas preconizadas como também em relação ao seu papel enquanto 
mediadores do processo de mudança à atenção a TB.

Torna-se fundamental a realização de outros estudos, de modo a identificar e refletir sobre a organização da APS para o diagnóstico e tratamento da TB na visão dos gestores de saúde.

\section{REFERÊNCIAS}

1. World Health Organization (WHO). Global Tuberculosis Report. [Internet] WHO report 2016[acesso em $01 \mathrm{abr}$ 2017].Disponível: http://www.who.int/tb/publications/global_report/gtbr2016_executive_summary.pdf?ua=1.

2. Clementino FC, Marcolino EC, Gomes LB, Guerreiro JV, de Miranda FAN. Ações de controle da tuberculose: análise a partir do programa de melhoria do acesso e da qualidade da atenção básica. Texto Contexto Enferm. [Internet] 2016;25(4) [acesso em 02 abr 2017]. Disponível: http://dx.doi.org/10.1590/0104-07072016004660015.

3. Feitoza DS, Clares JWB, Rodrigues LV, de Almeida PC. Vigilância epidemiológica no contexto do programa de controle da tuberculose: limites e possibilidades. Rev. Rene. 2012;13(5):1066-74.

4. Brasil. Decreto n. 7508, de 28 de junho de 2011. Regulamenta a Lei no 8.080, de 19 de setembro de 1990, para dispor sobre a organização do Sistema Único de Saúde - SUS, o planejamento da saúde, a assistência à saúde e a articulação interfederativa. Diário Oficial da União, 29 jun 2011, Seção 1. p.1.

5. Brasil. Lei n. 8080, de 19 de setembro de 1990. Dispõe sobre as condições para a promoção, proteção e recuperação da saúde, a organização e o funcionamento dos serviços correspondentes e dá outras providências. Diário Oficial da União, 20 set 1990, Seção 1. p.18055.

6. Silva BFS, Benito GAV. A voz de gestores municipais sobre o acesso à saúde nas práticas de gestão. Ciênc. saúde coletiva. 2013;18(8):2189-2200.

7. Barrêto AJR, de Sá LD, Nogueira JA, Palha PF, Pinheiro PGOD, de Farias NMP, et al. Organização dos serviços de saúde e a gestão do cuidado à tuberculose. Ciênc. saúde coletiva. [Internet]2012;17(7) [acesso em 20 mai 2014]. Disponível: http://dx.doi.org/10.1590/S1413-81232012000700027.

8. Natal (RN). Secretaria Municipal de Saúde. Plano Municipal de Saúde 2014-2017 - Diretrizes, Metas e Objetivos. Natal (RN): Secretaria Municipal de Saúde; 2014.

9. Ministério da Saúde (BR). Secretaria de Vigilância em Saúde. Programa Nacional de Controle da Tuberculose. Brasília (DF): Ministério da Saúde; 2011.

10. Fundação de Amparo à Pesquisa do Estado de São Paulo (FAPESP). Projeto FAPESP Regular. Avaliação da integração de serviços de saúde para o tratamento da tuberculose na Atenção Primária nas redes de atenção em regiões do Brasil. São Paulo: FAPESP; 2013.

11. Ministério da Saúde (BR). Conselho Nacional de Saúde. Diretrizes e normas regulamentadoras de pesquisa envolvendo seres humanos. Resolução n. 466, de 12 de dezembro de 2012. Brasília; 2012.

12. Pinto ESG, de Menezes RMP, Villa TCS. Situação de trabalho dos profissionais da Estratégia Saúde da Família em Ceará-Mirim. Rev. esc. enferm. USP. 2010;44(3):657-64.

13. de Macedo SM, Andrade RPS, Souza CRBA, Andrade ASS, Villa TCS, Pinto ESG. Estratégias para capacitação ao cuidado em tuberculose. Cogitare Enferm. 2016;21(3):1-8.

14. Nunes EFPA, Carvalho BG, Nicoletto SCS, Cordoni Junior L. Trabalho gerencial em Unidades Básicas de Saúde de municípios de pequeno porte no Paraná, Brasil. Interface (Botucatu). [Internet] 2016;20(58)[acesso em 02 abr 2017]. Disponível: http://dx.doi.org/10.1590/1807-57622015.0065.

15. Ditterich RG, Moysés ST, Moysés SJ.O uso de contratos de gestão e incentivos profissionais do setor público de saúde. Cad. Saúde Pública. 2012;28(4):615-27.

16. de Moraes PN, Iguti AM. Avaliação do desempenho do trabalhador como forma peculiar de prescrição do trabalho: uma análise do PMAQ-AB.Saúde debate. [Internet] 2013;37(98)[acesso em 15 abr 2014].Disponível: http://dx.doi.org/10.1590/S0103-11042013000300005. 
17. Ministério da Saúde (Brasil). Resolução n. 5, de 19 de junho de 2013. Dispõe sobre as regras do processo de pactuação de Diretrizes, Objetivos, Metas e Indicadores para os anos de 2013 - 2015, com vistas ao fortalecimento do planejamento do Sistema Único de Saúde (SUS) e a implementação do Contrato Organizativo da Ação Pública da Saúde (COAP). Diário Oficial da União, 20 jun 2013, Seção 1. p.29.

18. Ministério da Saúde (Brasil). Manual de recomendações para o controle da tuberculose no Brasil. [Internet] Brasília:Ministério da Saúde; 2011 [acesso em 22 mar 2014]. Disponível:http://bvsms.saude.gov.br/bvs/publicacoes/ manual_recomendacoes_controle_tuberculose_brasil.pdf.

19. Ministério da Saúde (Brasil).Secretaria de Políticas de Saúde. Departamento de Atenção Básica. Cadernos de Atenção Básica. Manual técnico para o controle da Tuberculose.Brasília(DF): Ministério da saúde; 2011.

20. Natal (RN). Sistema de Informação de Agravos de Notificação (SINAN). Departamento de vigilância a saúde. Setor de vigilância epidemiológica.Natal: Núcleo de agravos notificáveis; 2012.

21. Palha PF, da Silva LMC, Wysocki AD, Andrade RLP, Protti ST, Scatena LM, et al. Acesso aos serviços de atenção à tuberculose:análise da satisfação dos doentes. Rev. esc. enferm. USP. 2012;46(2):342-8.

22. Possuelo LG, Reis AJ, da Rosa AW, Poli H, Battisiti F, Sartori MS, et al.Tuberculose: um estudo sobre referência em serviços de saúde.RevEpidemiolControllnfecct. 2012;2(3):89-93.

23. Dantas DNA, Enders BC, de Queiroz AAR, Coura AS, da Silva MPM, de Menezes RMP. Fatores associados à primeira escolha de local para o diagnóstico da tuberculose.Rev. GaúchaEnferm. 2014;35(3):75-81.

24. Silva Sobrinho RA, Andrade RLP, Ponce MAZ, Wysocki AD, Brunello ME, Scatena LM, et al. Retardo no diagnóstico da tuberculose em município da tríplice fronteira Brasil, Paraguai e Argentina. Rev. Panam. Salud Pública. 2012;31(6):461-8.

25. de Lima L, dePires DEP, Forte ECN, Medeiros F. Satisfação e insatisfação no trabalho de profissionais de saúde da atenção básica. Esc. Anna Nery. [Internet] 2014;18(1) [acesso em 10 mai 2014]. Disponível: http://dx.doi. org/10.5935/1414-8145.20140003.

26. Mendonça MHM, Martins MIC, Giovanella L, Escorel S. Desafios para gestão do trabalho a partir de experiências exitosas de expansão da Estratégia de Saúde da Família.Ciênc. saúde coletiva. 2010;15(5):2355-65.

27. Piva SGN, Costa MCN, Barreto FR, Pereira SM. Prevalência de deficiência nutricional em pacientes com tuberculose pulmonar. J. Bras. Pneumol. 2013;39(4):476-83.

28. Barbosa IR, Costa ICC. Aspectos epidemiológicos da tuberculose no município de Natal.RevEnferm UFPI. 2013;2(2):14-20. 